\title{
OBSTRUCTION THEORY IN 3-DIMENSIONAL TOPOLOGY: CLASSIFICATION THEOREMS
}

\author{
BY HARRIE HENDRIKS
}

Communicated by T. A. Chapman, January 24, 1977

We consider the classification up to homotopy of homotopy equivalences of compact 3-manifolds. Given two compact 3-manifolds (with base point and $C W$-decomposition) $(M, m)$ and $(N, n)$, in [3] we found an algebraic criterion for the existence of a degree 1 map $f:(M, \partial M) \longrightarrow(N, \partial N)$ extending a given map $f^{1}$ defined on the relative 1 -skeleton $(M, \partial M)^{1}$. Here we consider the space $H^{1}(M, m)$ of degree 1 homotopy equivalences $f:(M, m) \rightarrow(M, m)$ such that $f \mid \partial M=$ Id and $f$ is homotopic rel $\partial M \cup\{m\}$ to a map coinciding with the identity on $(M, \partial M)^{1}$. If $\partial M=\varnothing$, it is equivalent to say that $f$ induces the identity automorphism of $\pi_{1}(M, m)$. (If $\partial M \neq \varnothing$, we assume that $m \in \partial M$.) Important results are the following.

1. Following Waldhausen e.a. [6] a homotopy equivalence of $\mathbf{P}^{2}$-irreducible (closed) sufficiently large 3-manifolds is homotopic to a homeomorphism unique up to isotopy. Our result indicates that the exclusion of 2 -sided projective planes is necessary. Indeed, suppose $M$ is the connected sum of two nonsimply connected 3-manifolds, then we have

THEOREM ([2]). If $M$ contains 2-sided projective planes, $M$ admits a self homotopy equivalence, in $H^{1}(M, m)$, which is not homotopic to a homeomor. phism rel $\partial M$.

Recall that all elements of $H^{1}(M, m)$ are simple homotopy equivalences (in the sense of Whitehead).

On the other hand, let $S$ be an embedded 2-sphere in $M$ with collar $S \times$ $[0,1]$. Then the rotation along $S$ is the homeomorphism in $H^{1}(M, m)$ defined by the identity outside $S \times[0,1]$ and by a generator of $\pi_{1} S O(3)$ within $S \times[0,1]$.

TheOREM ([4]). Let $M$ be a 3-manifold which does not contain 2-sided projective planes, then every self homotopy equivalence in $H^{1}(M, m)$ is homotopic $\operatorname{rel} \partial M \cup\{m\}$ to a rotation along a sphere.

2. Let $R$ be the set of 2 -spheres $S$ in $M$ such that we can express $M=M_{1} \cup M_{2}$, where $M_{1} \cap M_{2}=S$, and where $M_{1} \cup_{S} D^{3}$ is a connected sum of closed manifolds, each either with finite fundamental group whose 2-Sylow

AMS (MOS) subject classifications (1970). Primary 55D10, 55G37, 57A10. 
subgroup is cyclic or homotopy equivalent to a $S^{\mathbf{2}}$ or $\mathrm{P}^{\mathbf{2}}$ fibration over $S^{\mathbf{1}}$.

THEOREM ([4]). The rotation along an embedded 2-sphere $S$ is homotopic to the identity rel $\partial M$ if and only if $S \in R$.

For nonseparating spheres $S$, this is contained in [5].

3. The composition in $H^{1}(M, m)$ defines a multiplication in $\pi_{0} H^{1}(M, m)$.

THEOREM. $\pi_{0} H^{1}(M, m)$ is a group of exponent 2 .

More completely, let $\Lambda=\left\{\lambda \in \pi_{1}(M, m) ; \lambda^{2}=e, \lambda\right.$ reverses the orientation \} . 1 By [1] there exists for each $\lambda \in \Lambda$ an immersion $\sigma_{\lambda}:\left(S^{2}, *\right) \rightarrow$ $(M, m)$ such that $\sigma_{\lambda}\left(S^{2}\right)$ is a (2-sided) projective plane carrying the loop $\lambda$. Let $W(\Lambda)$ denote the $Z_{2}$-module with generators $\Lambda \times \Lambda$ and relations $\langle\lambda, \mu\rangle=$ $\langle\mu, \lambda\rangle=\left\langle\xi \lambda \xi^{-1}, \xi \mu \xi^{-1}\right\rangle=\langle\lambda, \mu \lambda \mu\rangle$ for every $\lambda, \mu \in \Lambda$ and $\xi \in \pi_{1} M$. Let $R$ denote the $\pi_{1} M$ submodule of $\pi_{2} M$ generated by $R$.

MAIN Theorem ([4]). Suppose $\pi_{2} M \neq 0$. There is an exact sequence of $\mathbf{Z}_{2}$ modules:

$$
0 \rightarrow \mathrm{Z}_{2} \otimes_{\pi}\left(\pi_{2} M\right) / R \stackrel{r}{\rightarrow} \pi_{0} H^{1}(M, m) \rightarrow W(\Lambda) \oplus \mathrm{Z}_{2} \otimes_{\pi} \mathrm{Z}[\Lambda] \rightarrow 0,
$$

where $\otimes_{\pi}$ denotes the tensor product over $\mathbf{Z}\left[\pi_{1} M\right]$.

If $\sigma:\left(S^{2}, *\right) \rightarrow(M, m)$ is an embedding or an immersion with image a 2-sided projective plane, $r(1 \otimes \sigma)$ is represented by the rotation along $\sigma\left(S^{2}\right)$. Let $\lambda, \mu \in \Lambda$, and suppose $f: M \rightarrow M$ is a map different from the identity only in a 3-ball where it differs by $\sigma_{\lambda} \circ H o p f \in \pi_{3} M$, where Hopf denotes the Hopf fibration $S^{3} \rightarrow S^{2}$ (resp. by the Whitehead product $\left[\sigma_{\lambda}, \sigma_{\mu}\right]$ ). Then the arc component of $f$ is mapped to $1 \otimes \lambda$ (resp. $\langle\lambda, \mu\rangle)$.

\section{BIBLIOGRAPHY}

1. D. B. A. Epstein, Projective planes in 3-manifolds, Proc. London Math. Soc. (3) 11 (1961), 469-484. MR 27 \# 2968.

2. H. Hendriks, Une obstruction pour scinder une équivalence d'homotopie en di. mension 3, Ann. Sci. École. Norm. Sup. (4) 9 (1976), 437-467.

3. Obstruction theory in 3-dimensional topology: an extension theorem, Report 7617, Nijmegen (1976); J. London Math. Soc. (to appear).

4. - Applications de la théorie d'obstruction en dimension 3, available as Publ. math. d'Orday $N^{\circ} 133-7535$ (1975); also, Mémoire Soc. Math. France (to appear).

5. L. Pontryagin, A classification of mappings of the three-dimensional complex into the two-dimensional sphere, Réc. Math. [Mat. Sbornik] N. S. 9 (51) (1941), 331-363. MR 3, 60 .

6. G. P. Scott, On sufficiently large 3-manifolds, Quart. J. Math. Oxford Ser. (2) 23 (1972), 159-172; Correction, ibid (2) 24 (1973), 527-529. MR 52 \# 4295.

MATHEMATISCH INSTITUUT, KATHOLIEKE UNIVERSITEIT, NIJMEGEN, NEDERLAND

$1 \pi_{1}(M, m)$ acts on $\Lambda$ by conjugation. 